\title{
2021 ESC Guidelines \\ for the Diagnosis and \\ Treatment of Acute and \\ Chronic Heart Failure
}

Natasha Meunier-McVey

Editorial Assistant

Citation: EMJ Cardiol. 2021;9[1]:22-25.

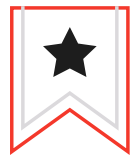

$\Lambda$ $n$ engaging session on the newly revised European Society of Cardiology (ESC) guidelines for the diagnosis and treatment of acute and chronic heart failure (HF)

was conducted on the first day of the 2021 ESC virtual congress. Chaired by Colin Baigent, Professor of Epidemiology, University of Oxford, UK, the session also explored the classification and management of HF, and addressed questions from the congress audience.

\section{THE MANAGEMENT OF HEART FAILURE WITH REDUCED EJECTION FRACTION}

Roy Gardner, Honorary Professor at the Institute of Cardiovascular and Medical Sciences, University of Glasgow, UK, opened the session by exploring the management of HF with reduced ejection fraction (HFrEF). Gardner outlined the key changes to the HFrEF therapeutic algorithm, last revised in 2016, which include the recommendation of dapagliflozin and empagliflozin for the management of HFrEF. These SGLT2 inhibitors have been given a Class IA recommendation under the new ESC guidelines, following the emergence of data from the DAPA-HF trial linking their use to reduced hospitalisation and mortality in patients with HF. The new 2021 therapeutic algorithm emphasises the importance of commencing key drug therapies as quickly and safely as possible following diagnosis.
These therapies include angiotensin-converting enzyme inhibitors, $\beta$-blockers, SGLT2 inhibitors, and mineralocorticoid receptor antagonists, and should be used in conjunction with a loop diuretic to relieve congestion.

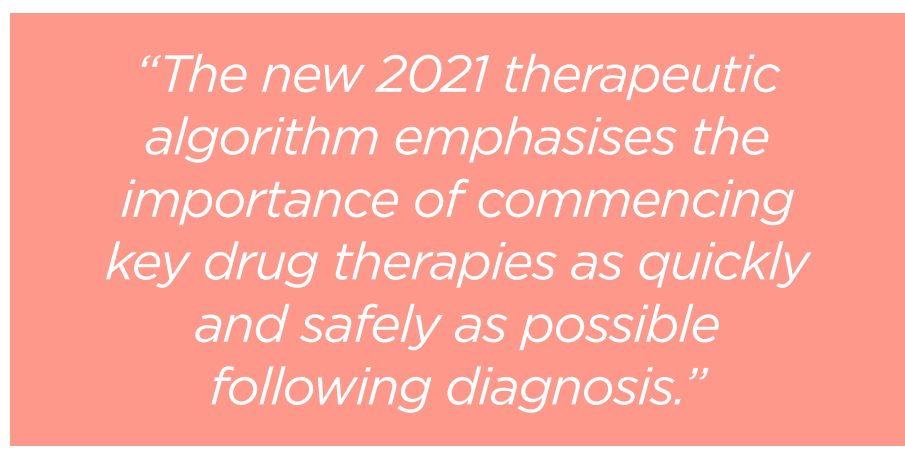

Gardner went on to explain the importance of tailored disease management in the $2021 \mathrm{HF}$ guidelines. The new guidelines advise clinicians to take a more phenotypic approach to the management of HFrEF, in an aim to reduce mortality through tailoring treatment to each 
patient. Aside from the disease-modifying therapies that can be prescribed, selected patients can also be offered device therapy, heart transplantation, or specific treatments for comorbidities. Device therapies for patients with advanced HF include mechanical circulatory support of various forms. Gardner also discussed methods to improve patient quality of life, such as exercise rehabilitation and multidisciplinary care.

\section{DIAGNOSIS AND TREATMENT OF HEART FAILURE WITH MILDLY REDUCED EJECTION FRACTION AND HEART FAILURE WITH PRESERVED EJECTION FRACTION}

Carolyn Lam, Professor of Duke-NUS Cardiovascular Academic Clinical Programme, Singapore, presented a session on the diagnosis and treatment of HF with mildly reduced ejection fraction (HFmrEF), and HF with preserved ejection fraction (HFpEF). Lam began by defining HF as "a clinical syndrome consisting of cardinal symptoms that may be accompanied by signs." The speaker then went on to explain the diagnostic algorithm of HF, which begins with the clinical assessment of these signs through ECG to determine underlying cardiac abnormalities. The left ventricular ejection fraction (LVEF) is then assessed for HF classification; an LVEF of $\leq 40 \%$ indicates HFrEF, 41-49\% HFmrEF, and $\geq 50 \%$ HFpEF.

One of the most notable changes from the 2016 guidelines in this area of HF is the modification of the term 'HF with midrange ejection fraction' to 'HF with mildly reduced ejection fraction'. This change was implemented following evidence demonstrating that patients with HFmrEF could benefit from similar therapies to those recommended for individuals with HFrEF. A table of recommendations for HFmrEF treatment has also been added to the guidelines, an area which was previously merged with HFpEF treatments.

Lam explained the challenges related to the diagnosis of HFpEF and outlined the guideline alterations implemented to improve this. The 2021 guidelines now outline a simplified approach to HF diagnosis, which focuses on the most widely

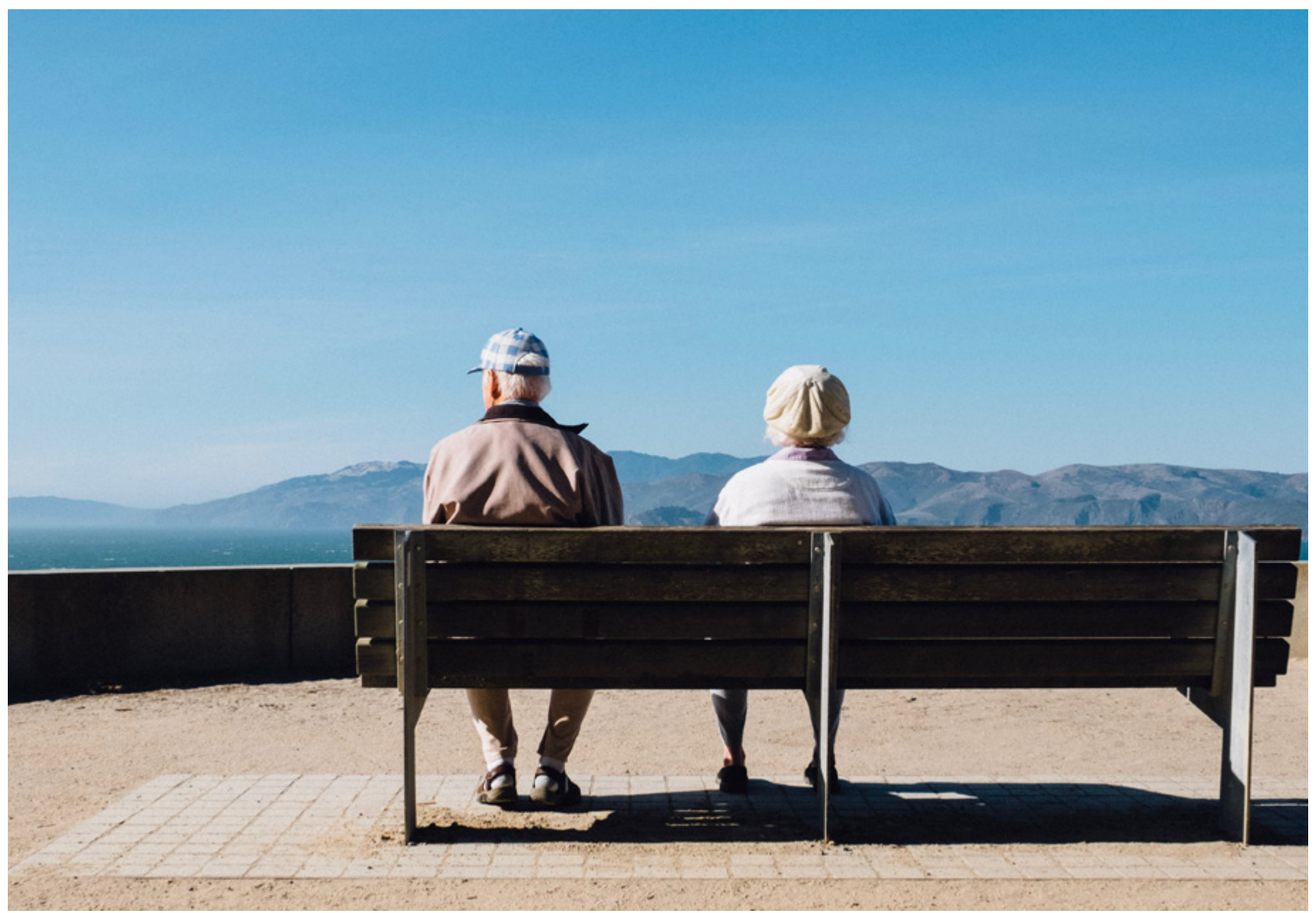


used and accessible diagnostic methods. Patients with HFrEF who experience an improvement in LVEF $(\geq 50 \%)$ are now described as having 'recovered HFrEF', rather than HFpEF. Although some improvements have been observed in some cases, no treatments have yet reduced mortality or morbidity in patients with HFpEF. Treatments are under ongoing revisions, and recommendations such as reducing body weight in obese patients continue to be advised where appropriate.

\section{NEW RECOMMENDATIONS FOR COMORBIDITIES}

Marianna Adamo, Interventional Cardiologist at the ASST Civil Hospital of Brescia, Italy, discussed the 2021 ESC guidelines for comorbidities associated with HF. Adamo began by outlining the management of cardiovascular comorbidities in HFrEF, including atrial fibrillation. The revised guidelines recommend anticoagulation therapy, treatment of triggers, and optimisation of HF therapies in all patients with atrial fibrillation and HFrEF. Further treatment strategies were also recommended depending on the individual case and stability of the patient. Adamo then explored therapies for chronic coronary syndrome and valvular heart disease, before discussing non-cardiovascular comorbidities. In patients with Type 2 diabetes mellitus and HF, SGLT2 inhibitors have been recommended to reduce hospitalisations, adverse cardiovascular events, and death. Other common comorbidities discussed included iron deficiency and cancer. Adamo also presented the new algorithms for the management of myocarditis and amyloidosis.

\section{ADVANCED AND ACUTE HEART FAILURE}

In the final presentation, Ovidiu Choincel, Professor at the Iliescu Institute for Emergency Cardiovascular Diseases, Bucharest, Romania, explored the guidelines for advanced and acute HF. A new definition for advanced HF was provided, which is now described as a "distinct clinical entity in the progression of heart failure." The revised ESC guidelines also outline specific criteria that must be met in order for a condition to be classed as advanced HF, and provide new recommendations for device therapies and heart transplants. For the first time, organisational issues related to patient referral in advanced HF centres have been addressed, and the criteria for referral defined. End-of-life care was also considered, with an emphasis on ensuring the best quality of life for patients.

"One of the most notable changes from the 2016 guidelines in this area of HF is the modification of the term 'HF with midrange ejection fraction' to 'HF with mildly reduced ejection fraction.

Acute HF has consistently been defined as a 'rapid onset' condition by past guidelines. Choincel explained how research has now identified that worsening symptoms may also appear gradually, causing a shift in definition of the disease. He went on to identify acute HF as a multi-event disease and presented a revised definition that characterises acute $\mathrm{HF}$ as "the rapid or gradual onset of symptoms of HF, severe enough to seek urgent medical attention and leading to an unplanned hospital admission or an emergency department visit." Results from clinical trials GALACTIC-HF and ELISABETH showed no benefit from early sustained vasodilation for those with HF, which led to a decrease in guideline classification for vasodilators. Although the classification for vasoconstrictors has remained the same, the revised guidelines have removed the recommendation of epinephrine following the OptimaCC trial, which presented an increase in refractory cardiogenic shock in patients treated with this drug. 


\section{CONCLUDING REMARKS}

to optimising patient care. Ongoing research and clinical trials are essential

These updated ESC guidelines will undoubtedly further help physicians in clinical decision-making and will contribute for ensuring a continuous improvement of clinical guidelines leading to better disease outcomes. 